\title{
The Pretreatment Controlling Nutritional Status Score in Ovarian Cancer: Influence on Prognosis, Surgical Outcome, and Postoperative Complication Rate
}

\author{
Der Controlling Nutritional Status Score vor Beginn \\ der Ovarialkarzinomtherapie: Bedeutung für Prognose, \\ Operationserfolg und postoperative Komplikationsrate
}

\section{(C) (1) $\Theta$}

Authors

Christine Bekos ${ }^{1}$, Christoph Grimm ${ }^{1}$, Lisa Gensthaler ${ }^{1,2}$, Thomas Bartl ${ }^{1}$, Alexander Reinthaller ${ }^{1,3}$, Richard Schwameis ${ }^{1}$, Stephan Polterauer ${ }^{1,3}$

\section{Affiliations}

1 Division of General Gynecology and Gynecologic Oncology, Department of Obstetrics and Gynecology, Gynecologic Cancer Unit, Comprehensive Cancer Center, Medical University of Vienna, Vienna, Austria

2 Department of Surgery, Medical University of Vienna, Vienna, Austria

3 Karl Landsteiner Institute for General Gynecology and Experimental Gynecologic Oncology, Vienna, Austria

Key words

ovarian cancer, ovarian neoplasms, malabsorption

syndromes, postoperative complications

\section{Schlüsselwörter}

Ovarialkarzinom, ovarielle Neoplasie, Malabsorptions-

syndrome, postoperative Komplikationen

\section{received}

accepted after revision

\section{Bibliography}

Geburtsh Frauenheilk 2022; 82: 59-67

DOI 10.1055/a-1608-1309

ISSN 0016-5751

(c) 2022. The Author(s).

This is an open access article published by Thieme under the terms of the Creative Commons Attribution-NonDerivative-NonCommercial-License, permitting copying and reproduction so long as the original work is given appropriate credit. Contents may not be used for commercial purposes, or adapted, remixed, transformed or built upon. (https://creativecommons.org/licenses/by-nc-nd/4.0/)

Georg Thieme Verlag KG, Rüdigerstraße 14,

70469 Stuttgart, Germany
Correspondence

Christoph Grimm, MD

Associate Professor, Medical University of Vienna

Währinger Gürtel 18-20, 1090 Vienna, Austria

christoph.grimm@meduniwien.ac.at

\section{ABSTRACT}

Introduction The Controlling Nutritional (CONUT) Status score is an established predictor of impaired prognosis in patients with solid tumors. The aim of this study was to investigate the prognostic value of the CONUT score for overall survival and perioperative complication rates in patients with epithelial ovarian cancer.

Patients In this retrospective study we assessed the data of 337 consecutive patients with ovarian cancer. The CONUT score was associated with surgical outcome, postoperative complications and clinicopathological parameters. We used univariate log-rank test and multivariable Cox regression models to evaluate the association between pretreatment CONUT scores and survival.

Results A low CONUT score (0-2) was associated with an early FIGO stage $(p=0.004)$, complete tumor resection $(p<0.001)$, less neoadjuvant chemotherapy $(p=0.017)$ and other histologies than serous cystadenocarcinoma $(p=0.006)$. Postoperative complications were observed in $51.4 \%$ and $60.5 \%$ of patients with a CONUT score of $0-2$ and a score $>2$, respectively $(p=0.161)$. A shorter overall survival was observed in patients with a CONUT score $>2$ compared to patients with a low CONUT score, with 5-year overall survival rates of $31.5 \%$ and $58.7 \%$, respectively $(p<0.001)$. In multivariable analysis, both advanced age $(p<0.001)$ and FIGO stage $(p<0.001)$, residual disease $(p<0.001)$ and a high CONUT score $(p=0.048)$ were independently associated with unfavorable overall survival. 
Conclusion Pretreatment CONUT score is an independent prognostic marker for overall survival and associated with successful surgery. Patients with a high CONUT score might benefit from pretreatment nutritional intervention.

\section{ZUSAMMENFASSUNG}

Einleitung Der Controlling Nutritional (CONUT) Status Score ist ein etablierter Prädiktor für eine eingeschränkte Prognose bei Patienten mit soliden Tumoren. Ziel dieser Studie war es, den prognostischen Wert des CONUT-Scores für das Gesamtüberleben und für perioperative Komplikationsraten bei Patientinnen mit epithelialem Ovarialkarzinom zu untersuchen.

Patientinnen In dieser retrospektiven Studie wurden die Daten von 337 konsekutiv aufgenommenen Patientinnen mit Ovarialkarzinom analysiert. Der CONUT-Score war mit dem Operationsergebnis, den postoperativen Komplikationen sowie den klinisch-pathologischen Parametern assoziiert. Wir verwendeten univariate Log-Rank-Tests sowie multivariable Cox-Regressionsmodelle, um die Assoziation zwischen dem CONUT-Score vor Beginn der Behandlung und das Überleben zu evaluieren.
Ergebnisse Ein niedriger CONUT-Score (0-2) war mit einem frühen FIGO-Stadium ( $p=0,004)$, einer vollständigen Resektion des Tumors $(p<0,001)$, weniger neoadjuvanter Chemotherapie $(p=0,017)$ und Tumoren, die keine seröse Zystadenokarzinome waren $(p=0,006)$, assoziiert. Postoperative Komplikationen traten bei $51,4 \%$ bzw. $60,5 \%$ von Patientinnen mit einem CONUT-Score von 0-2 bzw. einem Score von $>2$ auf $(p=0,161)$. Patientinnen mit einem CONUT-Score von >2 hatten ein kürzeres Gesamtüberleben verglichen mit Patientinnen mit einem niedrigen CONUT-Score, mit einer 5-Jahres-Überlebensrate von 31,5\% bzw. 58,7\% für die jeweilige Gruppe $(p<0,001)$. Die multivariable Analyse ergab, dass fortgeschrittenes Alter $(p<0,001)$, FIGO-Stadium $(p<0,001)$, Resterkrankung $(p<0,001)$ und hoher CONUTScore $(p=0,048)$ unabhängig voneinander mit ungünstigen Überlebensraten assoziiert waren.

Schlussfolgerungen Der CONUT-Score vor Beginn der Behandlung ist ein unabhängiger prognostischer Marker für das Gesamtüberleben und ist mit dem Operationserfolg assoziiert. Patientinnen mit einem hohen CONUT-Score könnten von einer Ernährungsintervention vor Beginn der Behandlung profitieren.

\section{Introduction}

Ovarian cancer is a rare disease but still represents the fifth most common malignancy in women [1]. Moreover, the five-year survival prognosis is poor, due to late diagnosis at advanced stages. Because of the absence of early symptoms and lack of an effective screening option, the majority of epithelial ovarian cancer (EOC) cases are diagnosed at an advanced stage. Various prognostic parameters have been evaluated for ovarian cancer, but none of them have entered clinical practice to date [2-5].

There is accumulating evidence on the impact of nutrition and inflammation status on mortality and morbidity in ovarian cancer [6-8]. Several biomarkers reflecting nutrition and inflammation status such as prognostic nutritional index [9], neutrophil-to-lymphocyte ratio [10], lymphocyte-to-monocyte ratio [11], plateletto-lymphocyte ratio [12] and the Glasgow prognostic score [13] have been identified as independent predictors of survival in patients with ovarian cancer.

The Controlling Nutritional Status (CONUT) score is a novel tool which evaluates nutritional and immunologic status. It is calculated using serum albumin concentration, total lymphocyte count and cholesterol concentration [14]. A high CONUT score reflects malnutrition and immunoactivation and is associated with a poor prognosis [14].

The CONUT score has been reported to be a prognostic survival marker for several cancers [15-18]. To our knowledge, only one study has investigated the significance of the CONUT score in ovarian cancer patients [19]. In 2020, Li et al. demonstrated in a small cohort that the CONUT score is an independent prognostic factor for overall survival. Although malnutrition has been linked to postoperative complications in ovarian cancer patients [2], the above-mentioned study failed to evaluate the impact of a preoperative CONUT score on postoperative complications.

The aim of this study was to investigate the value of the CONUT score as an indicator for a poor prognosis and the association between CONUT score and postoperative complications in a large cohort of epithelial ovarian cancer patients treated at a cancer center.

\section{Materials and Methods}

Three hundred and thirty-seven consecutive patients treated for epithelial ovarian cancer between 2000 and 2015 at the Comprehensive Cancer Center Vienna at the Medical University of Vienna were enrolled. Data were retrospectively extracted from paperbased and electronic medical records. As suggested in the current guidelines [20], tubal and primary peritoneal carcinomas were included in this patient cohort.

Patients were treated according to the standards of our institution, with upfront surgery and adjuvant platinum-based chemotherapy. Surgical staging according to FIGO guidelines was performed, including hysterectomy, bilateral salpingo-oophorectomy, pelvic and/or para-aortic lymphadenectomy, appendectomy, omentectomy, and a cytoreductive procedure to resect all gross tumor masses. Residual tumor load was defined as negative if macroscopically absent. Experienced gynecologic oncologists and an experienced pathologist performed the clinical and histopathologic evaluation.

Fertility-sparing surgery was performed in accordance with the patient's choice in young patients with ovarian tumors of low malignant potential or nonepithelial ovarian cancers and patients with stage IA EOC. If unilateral salpingo-oophorectomy was considered, full surgical staging, including washings, omentectomy, 
pelvic and paraaortic lymphadenectomy, and peritoneal biopsies were performed. Thorough abdominal exploration with biopsy of any abnormal areas was done. Endometrial biopsy to exclude synchronous endometrial cancer was completed.

NACT was given to patients with advanced EOC and unresectable disease who were unlikely to achieve complete cytoreduction if operated on at the time of presentation or patients with EOC who were initially poor candidates for surgery but were likely to tolerate surgery after chemotherapy.

For those who were unlikely to tolerate surgery at any point due to poor functional status, chemotherapy alone was administered for tumor control.

We assessed treatment response using computed tomography after three cycles of NACT. All patients were also seen by our interdisciplinary tumor board to determine whether to proceed with surgery. We proceeded with surgical cytoreduction in patients who did not progress during NACT and in whom there was a chance of resecting all residual disease or achieving optimal cytoreduction. After surgery, another three to six more cycles of chemotherapy were administered.

Patients with disease progression at any point, as well as those who showed an insufficient response after three cycles of therapy for optimal cytoreduction to be feasible, represent a group with chemoresistant disease. These patients were treated similarly to women with platinum-resistant EOC and typically did not pursue surgical options. However, in some patients, especially those with incomplete response, further rounds of neoadjuvant treatment were administered followed by re-evaluation for surgery of those who showed some response to NACT.

All treatment decisions were based on the conclusions of our weekly tumor board, which consisted of experienced gynecologic oncologists, a radiologist, a radiotherapist, an internal oncologist and an experienced pathologist.

Prior to treatment, a daily routine physical examination of patients was done by a specialist in internal medicine.

Patients with signs of active inflammation or chronic liver disease were excluded from the study.

Overall survival was the interval between diagnosis and cancerassociated death.

\section{Laboratory measurements}

Fasting blood collection for the evaluation of serum albumin, total lymphocyte count and total cholesterol concentration was performed by peripheral venous puncture during routinely performed blood tests prior to treatment.

CONUT scores were calculated using data for serum albumin, total cholesterol concentrations, and total peripheral lymphocyte counts [14]: (1) albumin concentrations $\geq 35$, 30-34.9, 25-29.9, and $<25 \mathrm{~g} / \mathrm{L}$ were scored as $0,2,4$, and 6 points, respectively; (2) total lymphocyte counts $\geq 1600,1200-1599$, 800-1199, and $<800 / \mathrm{mm}^{3}$ were scored as $0,1,2$, and 3 points, respectively; and (3) total cholesterol concentrations $\geq 180,140-179,100-139$, and $<100 \mathrm{mg} / \mathrm{dL}$ were scored as $0,1,2$, and 3 points, respectively. The CONUT score was defined as the sum of (1), (2), and (3). The cut-off for the CONUT score was $0-2$ and $>2$ as reported before [21].

\section{Patient follow-up}

Postoperative complications were graded according to the Clavien-Dindo classification [22].

The continuous follow-up of patients was documented until hospital discharge. Thereafter, patients were followed up every three months in the first year, every 6 months until the fifth year and annually up to 10 years. If patients missed scheduled followup visits, they were contacted by administrative personnel or nurses for follow-up data.

Complications which arose after hospital discharge were assessed in our outpatient clinic.

Residual tumor load was defined as negative if macroscopically absent. Overall survival was the interval between diagnosis and cancer-associated death. Patients without recurrence, cancer progression, or death were censored at the time of last follow-up.

Management of disease relapse depended on various criteria such as tumor biology, the patient's general condition (ECOG), toxicity, previous chemotherapy, and response to chemotherapy [23].

\section{Statistical analysis}

Values are presented as mean values with standard deviation. Student's t-test was performed to compare mean serum albumin levels with clinicopathological parameters. P-values of $<0.05$ were considered statistically significant. With respect to overall survival, differences between groups were evaluated using log-rank test and presented as Kaplan-Meier survival curves. Multivariable analysis was performed using a Cox regression model and included the CONUT score (> 2 vs. $0-2$ ), patient age (dichotomized at the median value of 59.5 years), tumor stage (FIGO IV. vs. FIGO III vs. FIGO II vs. FIGO I), tumor grade (G3 vs. G1/2) histology (serous adenocarcinoma vs. other) and residual disease (no residual disease vs. residual disease) as independent variables. Statistical analyses were performed using the statistical software SPSS version 25.0 .

\section{Results}

\section{Patient characteristics}

Patient demographics are shown in $>$ Table $\mathbf{1}$.

$67.1 \%$ of patients were treated with upfront surgery and adjuvant platinum-based chemotherapy. Surgical staging according to FIGO guidelines was performed, including hysterectomy, bilateral salpingo-oophorectomy, pelvic and/or para-aortic lymphadenectomy, appendectomy, omentectomy, and cytoreductive procedure to resect all gross tumor masses. Surgical resection without adjuvant treatment was performed in $16.0 \%$. Neoadjuvant chemotherapy with intervention debulking surgery was performed in $10.4 \%$. Neoadjuvant chemotherapy with intervention debulking surgery and adjuvant chemotherapy was performed in $3.0 \%$. Neoadjuvant chemotherapy was administered in $0.6 \%$. Other standard forms of treatment were performed in $3 \%$ of patients.

BRCA status was available for 45 patients. Fifteen patients had a pathogenic BRCA1 mutation and 4 patients a pathogenic BRCA2 mutation. One patient had a somatic BRCA1 mutation and 3 patients a BRCA2 variant of unknown significance. Twenty-two out of 45 were BRCA1/2 wildtype patients. 
- Table 1 Patient characteristics of 337 patients with epithelial ovarian cancer.

\begin{tabular}{|c|c|}
\hline Parameter & n (\%) or median (IR) \\
\hline Total number of patients enrolled & 337 \\
\hline Age at diagnosis, years & 59.54 (range: 13.40 ) \\
\hline BMI & 25.84 (range: 9.66) \\
\hline \multicolumn{2}{|l|}{$\begin{array}{l}\text { Eastern Cooperative Oncology Group (ECOG) } \\
\text { status }\end{array}$} \\
\hline .0 & $266(78.9 \%)$ \\
\hline$\cdot 1$ & $31(9.2 \%)$ \\
\hline .2 & $6(1.8 \%)$ \\
\hline .3 & $1(0.3 \%)$ \\
\hline .4 & 0 \\
\hline - unknown & $33(9.8 \%)$ \\
\hline \multicolumn{2}{|l|}{ Histological type } \\
\hline - serous adenocarcinoma & $212(62.9 \%)$ \\
\hline - endometroid adenocarcinoma & $55(16.3 \%)$ \\
\hline " mucinous adenocarcinoma & $16(4.7 \%)$ \\
\hline - clear cell carcinoma & $16(4.7 \%)$ \\
\hline - other & $38(11.3 \%)$ \\
\hline \multicolumn{2}{|l|}{ Histological grade } \\
\hline . G1 & $48(14.2 \%)$ \\
\hline - G2 & $83(24.6 \%)$ \\
\hline. $\mathrm{G3}$ & $189(56.1 \%)$ \\
\hline - unknown & $11(3.3 \%)$ \\
\hline \multicolumn{2}{|l|}{ Tumor stage } \\
\hline - FIGOI & $70(20.8 \%)$ \\
\hline - FIGOII & $23(6.8 \%)$ \\
\hline - FIGO III & $194(57.6 \%)$ \\
\hline - FIGOIV & $49(14.5 \%)$ \\
\hline - unknown & $1(0.3 \%)$ \\
\hline \multicolumn{2}{|l|}{ Neoadjuvant chemotherapy } \\
\hline " yes & $47(13.9 \%)$ \\
\hline " no & $290(86.1 \%)$ \\
\hline \multicolumn{2}{|l|}{ Residual disease } \\
\hline - no residual disease & $207(61.4 \%)$ \\
\hline - residual disease & $123(36.5 \%)$ \\
\hline - unknown & $7(2.1 \%)$ \\
\hline \multicolumn{2}{|l|}{ Recurrence status } \\
\hline - No. of patients with recurrent disease & $202(59.9 \%)$ \\
\hline Median time to recurrent disease (months) & $17.0(8.0-34.75)$ \\
\hline \multicolumn{2}{|l|}{ Status at last observation } \\
\hline $\begin{array}{l}\text { alive with no evidence of disease or stable } \\
\text { disease }\end{array}$ & $143(42.4 \%)$ \\
\hline " progressive disease & $41(12.2 \%)$ \\
\hline - tumor-related death & $138(40.9 \%)$ \\
\hline - death from other causes & $15(4.5 \%)$ \\
\hline Median time of follow-up (months) & $28.0(14.0-71.0)$ \\
\hline
\end{tabular}

\section{CONUT score and postoperative complications}

In the present study, 256 (76.0\%) patients had a CONUT score of $0-2$ and $81(24.0 \%)$ patients had a CONUT score of $>2$. Calculated Controlling Nutritional Status scores broken down by clinicopathological parameters are provided in $>$ Table 2.

Advanced tumor stage, higher rate of residual disease after surgery, more serous cancers and more patients receiving neoadjuvant chemotherapy were observed in the group of patients with a CONUT score of $>2$.

Postoperative complications (mainly mild complications) were observed in $51.4 \%$ and $60.5 \%$ of patients with a CONUT score of $0-2$ and $>2$, respectively $(p=0.161)$. Severe complications (Clavien-Dindo classification 3-5) were observed in $10.5 \%$ and $14.8 \%$ of patients with a CONUT score of $0-2$ and $>2$, respectively. Any type of postoperative complication was observed in 176/337 (53.7\%) patients. Numbers of postoperative complications broken down by CONUT score are provided in $>$ Table 3.

- Table 2 Relationship between clinicopathological variables and Controlling Nutritional Status score in 337 patients with epithelial ovarian cancer.

\begin{tabular}{|c|c|c|c|}
\hline & $\begin{array}{l}\text { Controlling } \\
\text { Nutritional } \\
\text { Status score } \\
0-2(n=256)\end{array}$ & $\begin{array}{l}\text { Controlling } \\
\text { Nutritional } \\
\text { Status score } \\
>2(n=81)\end{array}$ & p-value ${ }^{1}$ \\
\hline Tumor stage & & & 0.004 \\
\hline - FIGOI & $62(24.3 \%)$ & 8 (9.9\%) & \\
\hline - FIGOII & $21(8.2 \%)$ & $2(2.5 \%)$ & \\
\hline - FIGO III & $135(52.9 \%)$ & $59(72.8 \%)$ & \\
\hline - FIGOIV & 37 (14.5\%) & $12(14.8 \%)$ & \\
\hline Residual disease & & & $<0.001$ \\
\hline - no residual disease & $172(68.8 \%)$ & 37 (46.3\%) & \\
\hline - residual disease & $78(31.2 \%)$ & $43(53.8 \%)$ & \\
\hline $\begin{array}{l}\text { Neoadjuvant } \\
\text { chemotherapy }\end{array}$ & & & 0.017 \\
\hline - yes & $29(11.3 \%)$ & $18(22.2 \%)$ & \\
\hline - no & 227 (88.7\%) & $63(77.8 \%)$ & \\
\hline Age at first diagnosis & & & 0.315 \\
\hline - $\leq 59.5$ years & $127(49.6 \%)$ & 35 (43.2\%) & \\
\hline - > 59.6 years & $129(50.4 \%)$ & $46(56.8 \%)$ & \\
\hline Histological grade & & & 0.117 \\
\hline - G1 & $42(16.7 \%)$ & $6(7.5 \%)$ & \\
\hline - G2 & $65(25.9 \%)$ & $18(22.5 \%)$ & \\
\hline - G3 & $137(54.6 \%)$ & $52(27.5 \%)$ & \\
\hline - unknown & $7(2.8 \%)$ & $4(5.0 \%)$ & \\
\hline Histological type & & & 0.006 \\
\hline $\begin{array}{l}\text { - serous cystadeno- } \\
\text { carcinoma }\end{array}$ & $153(59.8 \%)$ & $59(72.8 \%)$ & \\
\hline - other & $103(40.2 \%)$ & $22(27.2 \%)$ & \\
\hline
\end{tabular}




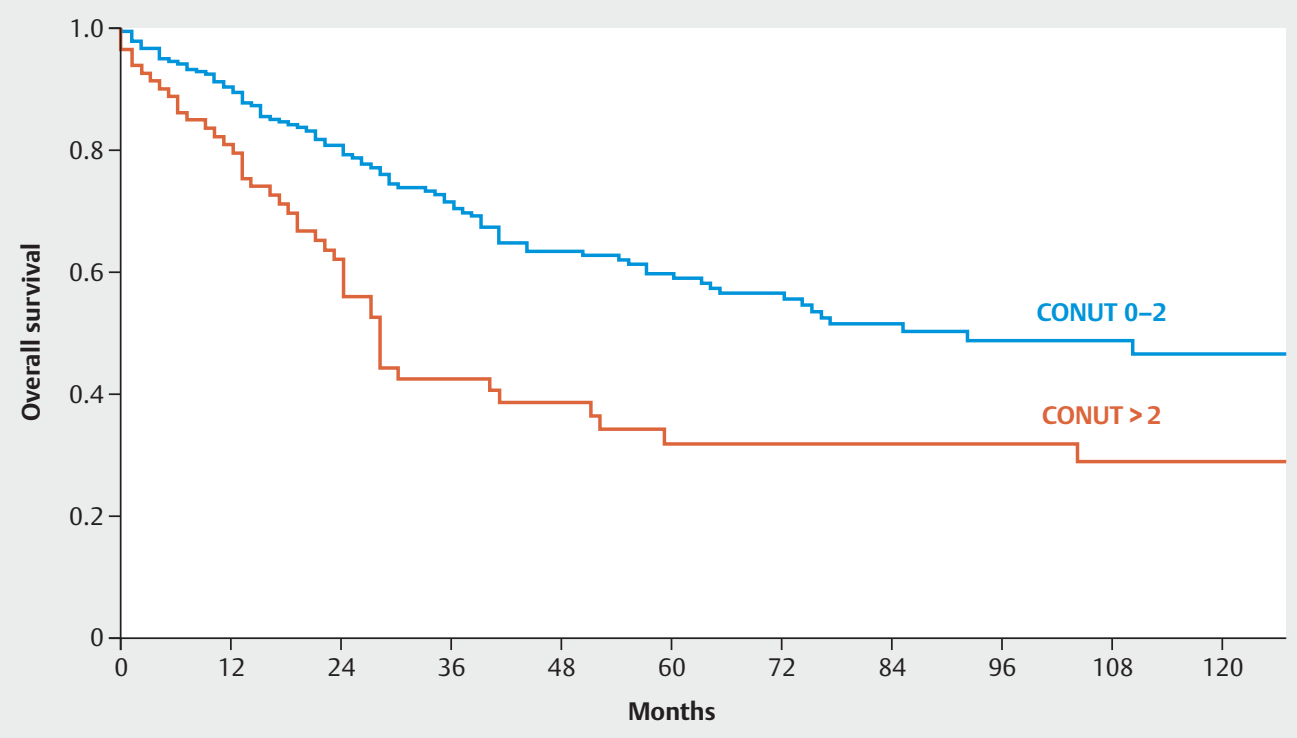

- Fig. 1 Kaplan-Meier curves for overall survival broken down according to pretreatment CONUT scores $(\mathrm{n}=337)$.

- Table 3 Numbers of postoperative complications classified according to the Clavien-Dindo classification and broken down according to the preoperative Controlling Nutritional Status score in 337 patients with epithelial ovarian cancer.

\begin{tabular}{|l|c|l|c|}
\hline $\begin{array}{l}\text { Postoperative } \\
\text { complications }\end{array}$ & $\begin{array}{l}\text { Controlling } \\
\text { Nutritional } \\
\text { Status score } \\
\mathbf{0 - 2}(\mathbf{n}=\mathbf{2 5 6})\end{array}$ & $\begin{array}{l}\text { Controlling } \\
\text { Nutritional } \\
\text { Status score } \\
\mathbf{2}(\mathbf{n}=\mathbf{8 1})\end{array}$ & $\begin{array}{l}\text { Complete } \\
\text { cohort }\end{array}$ \\
\hline None & $120(46.9 \%)$ & $32(39.5 \%)$ & $152(45.1 \%)$ \\
\hline Grade 1 & $34(13.3 \%)$ & $13(16.0 \%)$ & $47(13.9 \%)$ \\
\hline Grade 2 & $64(29.7 \%)$ & $24(29.6 \%)$ & $88(26.1 \%)$ \\
\hline Grade 3a & $5(2.0 \%)$ & $1(1.2 \%)$ & $6(1.8 \%)$ \\
\hline Grade 3b & $12(4.7 \%)$ & $9(11.1 \%)$ & $21(6.2 \%)$ \\
\hline Grade 4 & $8(3.1 \%)$ & $0(0.0 \%)$ & $8(2.4 \%)$ \\
\hline Grade 5 & $4(1.6 \%)$ & $2(2.5 \%)$ & $6(1.8 \%)$ \\
\hline Unknown & $9(3.5 \%)$ & $0(0.0 \%)$ & $9(2.8 \%)$ \\
\hline Total & 256 & 81 & 337 \\
\hline n= number & & & \\
\hline
\end{tabular}

\section{CONUT score and overall survival analysis}

The 5-year overall survival rate of patients with a CONUT score of $>2$ was $24.9 \%$ compared to $52.5 \%$ for patients with a CONUT score of $0-2,(p<0.001)$. The Kaplan-Meier survival curves in - Fig. 1 demonstrate the association between CONUT score and overall survival.

In univariate survival analysis, a high CONUT score $(p<0.001)$, advanced FIGO stage $(p<0.001)$, advanced patient age $(p<0.001)$, high tumor grade $(p=0.012)$, residual disease $(p<0.001)$, and serous histology $(p=0.022)$ were associated with poor overall survival. In multivariable analysis, a high CONUT score $(p=0.048)$, advanced FIGO stage $(p<0.001)$, advanced patient age $(p<0.001)$, and residual disease $(p<0.001)$ were associated with poor overall survival. Univariate and multivariate overall survival analyses are shown in $>$ Table 4.

\section{CONUT score and progression-free survival}

The 5-year progression-free survival rate of patients with a CONUT score $>2$ was $23.9 \%$ compared to $34.2 \%$ for patients with a CONUT score of $0-2(p=0.004)$.

The Kaplan-Meier survival curves in $\mathbf{F}$ Fig. 2 demonstrate the association between CONUT score and progression-free survival.

In univariate survival analysis, a high CONUT score $(p=0.004)$, advanced FIGO stage $(p<0.001)$, advanced patient age $(p=0.005)$, high tumor grade $(p<0.001)$, residual disease $(p<0.001)$, and serous histology $(p=0.002)$ were associated with poor progression-free survival. In multivariable analysis, advanced FIGO stage $(p<0.001)$, advanced patient age $(p=0.002)$, and residual disease $(p<0.001)$ were associated with poor progressionfree survival. Univariate and multivariate overall survival analyses are shown in $>$ Table 5 .

\section{Discussion}

In the present study, we analyzed the predictive and prognostic value of preoperative CONUT scores on postoperative complications and survival in a large cohort of consecutive patients with epithelial ovarian cancer. Our results demonstrate that pretreatment CONUT scores can be used as an independent prognostic factor for overall survival.

This observation has also been reported for various other cancer types, e.g., prostate cancer [18], esophageal carcinoma [21], and soft tissue sarcomas [24]. Our findings seem biologically plausible and are supported by the results of other studies [19]. Several preoperative assessment tools have been developed to 


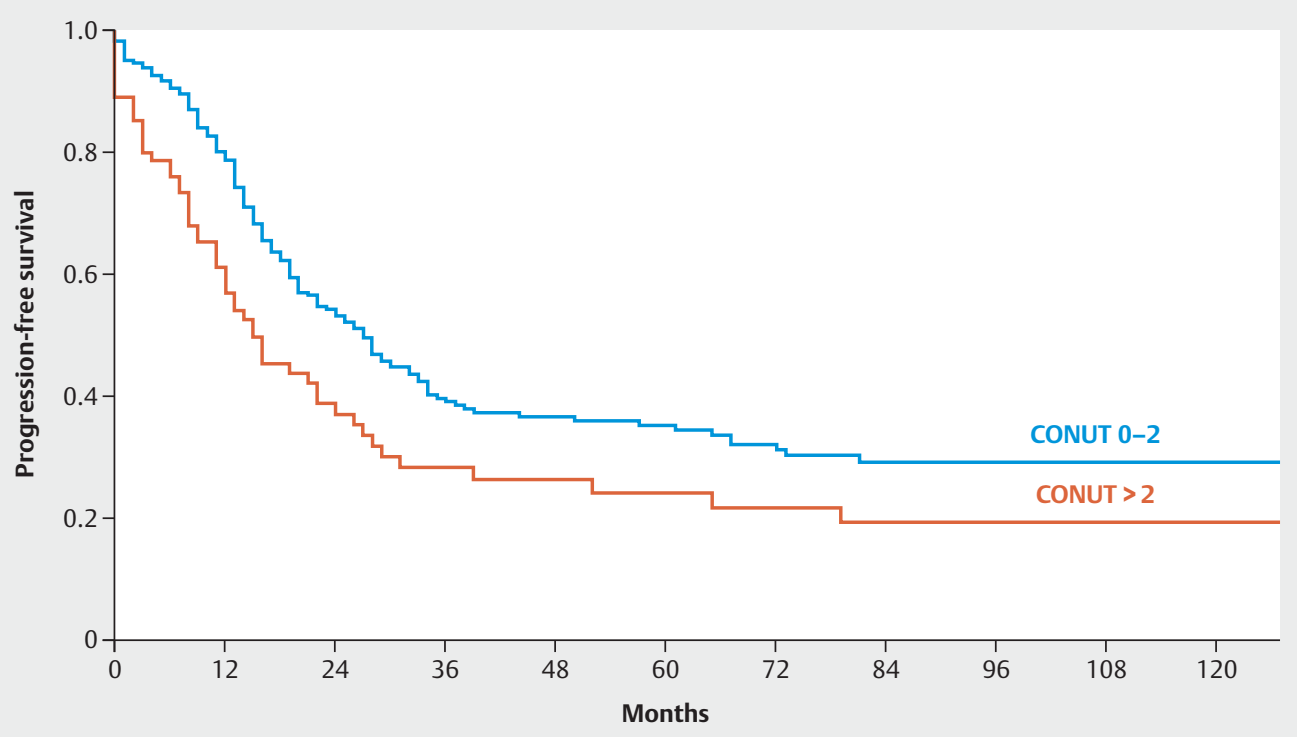

- Fig. 2 Kaplan-Meier curves for progression-free survival broken down according to pretreatment CONUT scores $(\mathrm{n}=337)$.

- Table 4 Univariate and multivariate overall survival analyses in 337 patients with epithelial ovarian cancer.

\begin{tabular}{|c|c|c|c|c|}
\hline & \multicolumn{4}{|c|}{ Overall survival } \\
\hline & \multicolumn{2}{|c|}{ Univariate $^{1}$ analysis } & \multicolumn{2}{|c|}{ Multivariate $^{2}$ analysis } \\
\hline & p-value & 5-year overall survival rate & p-value & $\mathrm{HR}(95 \% \mathrm{Cl})$ \\
\hline Controlling Nutritional Status score (> 2 vs. $0-2$ ) & $<0.001$ & $31.5 \%$ vs. $58.7 \%$ & 0.048 & $1.4(1.0-2.1)$ \\
\hline FIGO stage (IV vs. III vs. II vs. I) & $<0.001$ & $28.9 \%$ vs. $42.3 \%$ vs. $66.8 \%$ vs. $92.0 \%$ & $<0.001$ & $1.5(1.2-1.9)$ \\
\hline Patient age (> 59.6 vs. $\leq 59.5$ years) & $<0.001$ & $42.2 \%$ vs. $64.0 \%$ & $<0.001$ & $2.0(1.4-2.9)$ \\
\hline Histological grade (high-grade vs. low-grade) & 0.012 & $42.3 \%$ vs. $62.8 \%$ & 0.890 & $1.0(0.7-1.5)$ \\
\hline Residual disease (residual disease vs. no residual disease) & $<0.001$ & $23.8 \%$ vs. $66.7 \%$ & $<0.001$ & $2.5(1.7-3.6)$ \\
\hline Type of histology (serous adenocarcinoma vs. other) & 0.022 & $45.0 \%$ vs. $64.1 \%$ & 0.936 & $1.0(0.7-1.4)$ \\
\hline
\end{tabular}

- Table 5 Univariate and multivariate progression-free survival analyses in 337 patients with epithelial ovarian cancer.

\begin{tabular}{|c|c|c|c|c|}
\hline & \multicolumn{4}{|c|}{ Progression-free survival } \\
\hline & \multicolumn{2}{|c|}{ Univariate $^{1}$ analysis } & \multicolumn{2}{|c|}{ Multivariate $^{2}$ analysis } \\
\hline & p-value & 5-year progression-free survival rate & p-value & HR $(95 \% \mathrm{Cl})$ \\
\hline Controlling Nutritional Status score (> 2 vs. $0-2$ ) & 0.004 & $21.5 \%$ vs. $34.2 \%$ & 0.119 & $1.3(0.9-1.8)$ \\
\hline FIGO stage (IV vs. III vs. II vs. I) & $<0.001$ & $4.5 \%$ vs. $19.7 \%$ vs. $37.1 \%$ vs. $79.7 \%$ & $<0.001$ & $2.0(1.6-2.4)$ \\
\hline Patient age (> 59.6 vs. $\leq 59.5$ years) & 0.005 & $25.0 \%$ vs. $39.3 \%$ & 0.002 & $1.6(1.2-2.1)$ \\
\hline Histological grade (high-grade vs. low-grade) & $<0.001$ & $27.4 \%$ vs. $59.7 \%$ & 0.290 & $0.8(0.6-1.2)$ \\
\hline Residual disease (residual disease vs. no residual disease) & $<0.001$ & $9.3 \%$ vs. $44.5 \%$ & $<0.001$ & $2.0(1.4-2.7)$ \\
\hline Type of histology (serous adenocarcinoma vs. other) & 0.002 & $22.6 \%$ vs. $47.0 \%$ & 0.808 & $1.0(0.7-1.5)$ \\
\hline
\end{tabular}


evaluate nutritional risk, postoperative complications, and longterm outcomes, such as the prognostic nutritional index, subjective global assessment, and nutritional risk index [8, 25, 26]. Obesity is an independent risk factor which considerably increases the probability of developing various cancer types, but its relevance for disease risk and risk of cancer-specific mortality in epithelial ovarian cancer is clinically negligible [27]. The CONUT score summarizes measurements of serum albumin, total cholesterol, and total lymphocyte count.

The combination of the three components may provide a better reflection of the balance of nutritional status and enhance the ability to accurately predict the patient's general condition. In a study of 697 patients with gastric cancer, the CONUT score was superior as an independent predictor of outcome compared to body mass index, prognostic nutritional index and performance status [28]. Furthermore, the CONUT score is an easy-to-use, cost-effective method of objectively and comprehensively evaluating patients in hospital settings.

Serum albumin is used as the standard parameter to evaluate a patient's nutritional status and has been shown to be closely associated with the prognosis of ovarian cancer [29]. Cholesterol is known to be an indicator of a patient's caloric reserves [30], and an important component of the cell membrane has been linked to proliferation, metastasis, and the immune response of tumor cells [31]. Several studies have demonstrated that lipid metabolism plays a substantial role in the pathology of malignancies and correlates with the prognosis of various solid tumors [32].

Total lymphocyte count is an established indicator of cell-mediated immune status, including both acquired and adaptive immunities. The lymphocyte subsets CD4+ and CD8+ T cells, natural killer cells and B cells are closely associated with tumor immunity. Several studies have demonstrated that a reduced number of these cells are associated with impaired tumor immunity, resulting in cancer progression [33].

Taken together, a higher pretreatment CONUT score is not only an indicator of malnutrition but also of systemic inflammatory activity and might be a useful tool which could help us recognize a broader high-risk population and provide optimized therapy such as pretreatment nutrition intervention or physical therapy intervention. Poor preoperative physical fitness and nutritional status are linked to poor postoperative outcomes [34]. 50-80\% of cancer patients suffer from cancer cachexia due to catabolic effects of the tumor, abnormal metabolism of nutrients, physical obstruction of the gastrointestinal tract, reduced food intake due to pain, anxiety, and depression [35]. Malnutrition in cancer patients has been associated with negative outcomes, altering the immunological response and increasing the risk of infection while decreasing functional capacity, tolerance to treatment, and chances of survival $[36,37]$. Furthermore, malnourished patients tend to experience prolonged hospitalization which negatively affects their prognosis [38]. Therefore, identifying interventions to optimize preoperative nutritional status and fitness prior to major surgery is a priority.

A randomized controlled study compared immune-enhancing enteral nutrition with standard enteral feeding in oncologic gynecologic patients (32\% of ovarian cancer patients) after surgery and found decreased postoperative complications and reduced length of hospital stay but no significant differences in mortality rates [39]. The length of hospital stay of patients receiving early oral feeding was shorter than the hospital stay of patients receiving traditional oral feeding [40]. Furthermore, patients who received traditional oral feeding presented with significantly more postoperative complications than patients who received early oral feeding [40].

According to the Enhanced Recovery After Surgery (ERAS) guidelines for perioperative care in gynecologic oncology, maintenance of a regular diet within the first 24 hours after gynecologic oncology surgery is recommended [41]. A higher postoperative protein intake is also associated with earlier discharge [42]. Currently, there are no definitive guidelines for surgical patients with regard to protein needs; however, in the acute care setting, guidelines have recommended up to $2.0 \mathrm{~g}$ of protein $/ \mathrm{kg} /$ day and $25-$ $30 \mathrm{kcal} / \mathrm{kg} /$ day [43].

High-quality studies on prehabilitation including exercise and dietary interventions in gynecologic oncology patients are still lacking. A number of publications on colorectal surgery have shown that nutrition prehabilitation with and without exercise shortened the length of hospital stay by 2 days in a largely traditional surgical care setting [44]. A meta-analysis of prehabilitation interventions consisting of inspiratory muscle training, aerobic exercise, and/or resistance training found that prehabilitation decreased postoperative complications after intraabdominal operations in a traditional surgical care setting [45]. These results for colorectal surgery show that certain patients will benefit clinically from prehabilitation, but further work in the field of gynecologic oncology is needed.

We found that advanced tumor stage, residual disease after surgery, serous histology and neoadjuvant treatment regimens were associated with a CONUT score of $>2$. These findings appear biologically plausible as a high tumor burden fosters tumor necrosis factor, transforming growth factor and interleukin-6 secretion, which are related to cancer cachexia through direct interference with protein homeostasis in muscle cells. These cytokines promote proteasome-mediated protein catabolism in differentiated muscle cells [46], suppress muscle-cell differentiation [47] and induce excessive fatty acid oxidation in muscle cells [48].

Other reports on breast cancer and ovarian cancer patients also showed an association between advanced tumor stage and high CONUT score $[15,19]$. It remains unclear whether a high CONUT score is directly caused by tumor load and cytokine secretion or whether it is a secondary result of increased vascular permeability contributing to a shift of albumin from the intravascular sector towards the interstitium [49], and of comorbidities associated with cancer, such as sepsis, chronic liver or renal disease and gastrointestinal bleeding, which contribute to malnutrition and inflammation through various mechanisms [50].

Our data support the findings of Li et al. [19] that a high pretreatment CONUT score is associated with a poor prognosis in ovarian cancer patients. Their investigation included 206 ovarian cancer patients, and a high CONUT score was found be associated with high FIGO stage, high volumes of ascites on imaging, high concentrations of CA 125, and chemoresistance.

For other tumor entities, the benefit of using the CONUT score to evaluate postoperative complications remains controversial 
$[16,17,28]$. In our study cohort, we observed no differences in the incidence of postoperative complications between patients with a preoperative CONUT score of $>2$ compared to patients with a low CONUT score. We observed a total postoperative complication rate of $53.7 \%$, with severe complications (Clavien-Dindo classification 3-5) occurring in $11.6 \%$.

In a retrospective study by Ataseven et al. investigating a cohort of 309 patients with epithelial ovarian cancer, a total complication rate of $83.5 \%$ and severe complication rate of $25.2 \%$ were observed after cytoreductive surgery [2]. Although the patient cohort and clinicopathological parameters are comparable between both studies, differences in postoperative management such as following the ERAS protocol [51], prophylactic antibiotics, offering chewing gum, coffee or an early oral diet may explain the different results. Due to the retrospective design, the rate of postoperative complications may also have been underestimated in our cohort.

The strengths of the present study include the uniform singleinstitution approach to care and the completeness of clinical data. The main potential limitation of this study - which is typical for retrospective studies - is the lack of random patient assignment and patient selection. However, our data are in line with several series published so far regarding the prognostic impact of CONUT scores in epithelial ovarian cancer and other tumor types.

\section{Conclusion}

In conclusion, this study suggests that CONUT scores might be a useful prognostic biomarker of overall survival in women with ovarian cancer that is low cost and broadly available. Future prospective investigations are warranted to investigate the clinical applications of CONUT scores in ovarian cancer patients. The impact of preoperative nutrition on patients suffering from malnutrition and the influence on prognosis in patients with ovarian cancer undergoing cytoreductive surgery need to be further elucidated.

\section{Declarations}

Funding: Not applicable.

Ethics approval: The study was approved by the Ethics Committee of the Medical University of Vienna (1696/2017) before the study was initiated.

Consent to participate: Due to its retrospective design, the Ethics Committee waived the requirement to obtain specific informed consent from patients.

Consent for publication: not applicable.

Availability of data and material (data transparency): In accordance with the journal's guidelines, we will provide our data for the reproducibility of this study in other centers if requested.

Code availability: not applicable.

Authors' contributions: All authors contributed to the study conception and design. Material preparation, data collection and analysis were performed by Lisa Gensthaler, Thomas Bartl, Richard Schwameis and Alexander Reinthaller. The first draft of the manuscript was written by Christine Bekos, Christoph Grimm and Stephan Polterauer; all authors commented on previous versions of the manuscript. All authors read and approved the final manuscript.

\section{Conflict of Interest}

Christoph Grimm: Consultant: AstraZeneca, Celgene, MSD, PharmaMar, Roche, GSK/Tesaro, Vifor Pharma, Clovis; Speaker: Amgen, AstraZeneca, MSD, PharmaMar, Roche, GSK/Tesaro; Direct research funding: Meda Pharma, Roche Diagnostics, AstraZeneca.

Stephan Polterauer: Consultant: AstraZeneca, Celgene, MSD, PharmaMar, Roche, GSK/Tesaro, Vifor Pharma, Clovis; Speaker: Amgen, AstraZeneca, MSD, PharmaMar, Roche, GSK/Tesaro; Direct research funding: Meda Pharma, Roche Diagnostics, AstraZeneca. Alexander Reinthaller: Consultant: AstraZeneca, Celgene, MSD, PharmaMar, Roche, GSK/Tesaro, Vifor Pharma, Clovis; Speaker: Amgen, AstraZeneca, MSD, PharmaMar, Roche, GSK/Tesaro; Direct research funding: Meda Pharma, Roche Diagnostics, AstraZeneca.

References

[1] Torre LA, Bray F, Siegel RL et al. Global cancer statistics, 2012. CA Cancer J Clin 2015; 65: 87-108. doi:10.3322/caac.21262

[2] Ataseven B, du Bois A, Reinthaller A et al. Pre-operative serum albumin is associated with post-operative complication rate and overall survival in patients with epithelial ovarian cancer undergoing cytoreductive surgery. Gynecol Oncol 2015; 138: 560-565. doi:10.1016/j.ygyno.2015.07.005

[3] Lafleur J, Hefler-Frischmuth K, Grimm C et al. Prognostic Value of Serum Creatinine Levels in Patients with Epithelial Ovarian Cancer. Anticancer Res 2018; 38: 5127-5130. doi:10.21873/anticanres. 12834

[4] Reiser E, Aust S, Seebacher V et al. Preoperative C-reactive protein serum levels as a predictive diagnostic marker in patients with adnexal masses. Gynecol Oncol 2017; 147: 690-694. doi:10.1016/j.ygyno.2017.09.018

[5] Seebacher V, Aust S, D'Andrea D et al. Development of a tool for prediction of ovarian cancer in patients with adnexal masses: Value of plasma fibrinogen. PloS One 2017; 12: e0182383. doi:10.1371/journal. pone. 0182383

[6] Aust S, Knogler T, Pils D et al. Skeletal Muscle Depletion and Markers for Cancer Cachexia Are Strong Prognostic Factors in Epithelial Ovarian Cancer. PloS One 2015; 10: e0140403. doi:10.1371/journal.pone.0140403

[7] Kumar A, Torres ML, Cliby WA et al. Inflammatory and Nutritional Serum Markers as Predictors of Peri-operative Morbidity and Survival in Ovarian Cancer. Anticancer Res 2017; 37: 3673-3677. doi:10.21873/ anticanres. 11738

[8] Yim GW, Eoh K], Kim SW et al. Malnutrition Identified by the Nutritional Risk Index and Poor Prognosis in Advanced Epithelial Ovarian Carcinoma. Nutr Cancer 2016; 68: 772-779. doi:10.1080/01635581.2016.1159702

[9] Feng Z, Wen H, Ju X et al. The preoperative prognostic nutritional index is a predictive and prognostic factor of high-grade serous ovarian cancer. BMC Cancer 2018; 18: 883. doi:10.1186/s12885-018-4732-8

[10] Chen G, Zhu L, Yang Y et al. Prognostic Role of Neutrophil to Lymphocyte Ratio in Ovarian Cancer: A Meta-Analysis. Technol Cancer Res Treat 2018; 17: 1533033818791500. doi:10.1177/1533033818791500

[11] Gao XP, Liu YH, Liu ZY et al. Pretreatment lymphocyte-to-monocyte ratio as a predictor of survival among patients with ovarian cancer: a metaanalysis. Cancer Manag Res 2019; 11: 1907-1920. doi:10.2147/CMAR. S184970

[12] Tian C, Song W, Tian X et al. Prognostic significance of platelet-to-lymphocyte ratio in patients with ovarian cancer: A meta-analysis. Eur J Clin Invest 2018; 48: e12917. doi:10.1111/eci.12917

[13] Omichi C, Nakamura K, Haraga J et al. Glasgow prognostic score is an independent marker for poor prognosis with all cases of epithelial ovarian cancer. Cancer Med 2016; 5: 1074-1080. doi:10.1002/cam4.681

[14] Ignacio de Ulibarri J, Gonzalez-Madrono A, de Villar NG et al. CONUT: a tool for controlling nutritional status. First validation in a hospital population. Nutr Hosp 2005; 20: 38-45 
[15] Li W, Li M, Wang T et al. Controlling Nutritional Status (CONUT) score is a prognostic factor in patients with resected breast cancer. Sci Rep 2020; 10: 6633. doi:10.1038/s41598-020-63610-7

[16] Tokunaga R, Sakamoto Y, Nakagawa S et al. CONUT: a novel independent predictive score for colorectal cancer patients undergoing potentially curative resection. Int J Colorectal Dis 2017; 32: 99-106. doi:10.1007/s00384-016-2668-5

[17] Yoshida N, Baba Y, Shigaki H et al. Preoperative Nutritional Assessment by Controlling Nutritional Status (CONUT) is Useful to estimate Postoperative Morbidity After Esophagectomy for Esophageal Cancer. World J Surg 2016; 40: 1910-1917. doi:10.1007/s00268-016-3549-3

[18] Zhang W, Wu Y, Zhang Z et al. Controlling Nutritional Status score: A new prognostic indicator for patients with oligometastatic prostate cancer. Curr Probl Cancer 2019; 43: 461-470. doi:10.1016/j.currproblcancer.2019.02.001

[19] Li Y, Zhang C, Ji R et al. Prognostic significance of the controlling nutritional status (CONUT) score in epithelial ovarian cancer. Int J Gynecol Cancer 2020; 30: 74-82. doi:10.1136/ijgc-2019-000865

[20] Colombo N, Sessa C, Bois AD et al. ESMO-ESGO consensus conference recommendations on ovarian cancer: pathology and molecular biology, early and advanced stages, borderline tumours and recurrent disease. Int J Gynecol Cancer 2019. doi:10.1136/ijgc-2019-000308

[21] Toyokawa T, Kubo N, Tamura T et al. The pretreatment Controlling Nutritional Status (CONUT) score is an independent prognostic factor in patients with resectable thoracic esophageal squamous cell carcinoma: results from a retrospective study. BMC Cancer 2016; 16: 722. doi:10.1186/s12885-016-2696-0

[22] Dindo D, Demartines N, Clavien PA. Classification of surgical complications: a new proposal with evaluation in a cohort of 6336 patients and results of a survey. Ann Surg 2004; 240: 205-213

[23] Claussen C, Rody A, Hanker L. Treatment of Recurrent Epithelial Ovarian Cancer. Geburtshilfe Frauenheilkd 2020; 80: 1195-1204. doi:10.1055/ a-1128-0280

[24] Liang Y, Hou T, Que Y et al. Elevated Controlling Nutritional Status (CONUT) Score is Associated with Poor Long-term Survival in Patients with Low-grade Soft-tissue Sarcomas Treated with Surgical Resection. Clin Orthop Relat Res 2019; 477: 2287-2295. doi:10.1097| CORR.0000000000000767

[25] Kang M, Chang CT, Sung $\mathrm{HH}$ et al. Prognostic Significance of Pre- to Postoperative Dynamics of the Prognostic Nutritional Index for Patients with Renal Cell Carcinoma Who Underwent Radical Nephrectomy. Ann Surg Oncol 2017; 24: 4067-4075. doi:10.1245/s10434-017-6065-2

[26] Secker DJ, Jeejeebhoy KN. Subjective Global Nutritional Assessment for children. Am J Clin Nutr 2007; 85: 1083-1089. doi:10.1093/ajcn/ 85.4.1083

[27] Kolbl H, Bartl T. Obesity in Gynecologic Oncology. Geburtshilfe Frauenheilkd 2020; 80: 1205-1211. doi:10.1055/a-1124-7139

[28] Kuroda D, Sawayama H, Kurashige J et al. Controlling Nutritional Status (CONUT) score is a prognostic marker for gastric cancer patients after curative resection. Gastric Cancer 2018; 21: 204-212. doi:10.1007| s10120-017-0744-3

[29] Ge LN, Wang F. Prognostic significance of preoperative serum albumin in epithelial ovarian cancer patients: a systematic review and dose-response meta-analysis of observational studies. Cancer Manag Res 2018; 10: 815-825. doi:10.2147/CMAR.S161876

[30] Gadgil MD, Anderson CA, Kandula NR et al. Dietary patterns are associated with metabolic risk factors in South Asians living in the United States. J Nutr 2015; 145: 1211-1217. doi:10.3945/jn.114.207753

[31] Oliver MF. Serum cholesterol-the knave of hearts and the joker. Lancet 1981; 2: 1090-1095. doi:10.1016/s0140-6736(81)91286-1

[32] Ahn J, Lim U, Weinstein SJ et al. Prediagnostic total and high-density lipoprotein cholesterol and risk of cancer. Cancer Epidemiol Biomarkers Prev 2009; 18: 2814-2821. doi:10.1158/1055-9965.EPI-08-1248
[33] Gooden M], de Bock GH, Leffers $\mathrm{N}$ et al. The prognostic influence of tumour-infiltrating lymphocytes in cancer: a systematic review with metaanalysis. Br J Cancer 2011; 105: 93-103. doi:10.1038/bjc.2011.189

[34] Carli F, Zavorsky GS. Optimizing functional exercise capacity in the elderly surgical population. Curr Opin Clin Nutr Metab Care 2005; 8: 23-32. doi:10.1097/00075197-200501000-00005

[35] von Haehling S, Anker SD. Prevalence, incidence and clinical impact of cachexia: facts and numbers-update 2014. J Cachexia Sarcopenia Muscle 2014; 5: 261-263. doi:10.1007/s13539-014-0164-8

[36] Tisdale MJ. Mechanisms of cancer cachexia. Physiol Rev 2009; 89: 381 410. doi:10.1152/physrev.00016.2008

[37] Marin Caro MM, Laviano A, Pichard C. Nutritional intervention and quality of life in adult oncology patients. Clin Nutr 2007; 26: 289-301. doi:10.1016/j.clnu.2007.01.005

[38] Rodrigues CS, Lacerda MS, Chaves GV. Patient Generated Subjective Global Assessment as a prognosis tool in women with gynecologic cancer. Nutrition 2015; 31: 1372-1378. doi:10.1016/j.nut.2015.06.001

[39] Celik JB, Gezginc K, Ozcelik K et al. The role of immunonutrition in gynecologic oncologic surgery. Eur J Gynaecol Oncol 2009; 30: 418-421

[40] Minig L, Biffi R, Zanagnolo $V$ et al. Reduction of postoperative complication rate with the use of early oral feeding in gynecologic oncologic patients undergoing a major surgery: a randomized controlled trial. Ann Surg Oncol 2009; 16: 3101-3110. doi:10.1245/s10434-009-0681-4

[41] Nelson G, Bakkum-Gamez J, Kalogera E et al. Guidelines for perioperative care in gynecologic/oncology: Enhanced Recovery After Surgery (ERAS) Society recommendations-2019 update. Int J Gynecol Cancer 2019; 29: 651-668. doi:10.1136/ijgc-2019-000356

[42] Yeung SE, Hilkewich L, Gillis C et al. Protein intakes are associated with reduced length of stay: a comparison between Enhanced Recovery After Surgery (ERAS) and conventional care after elective colorectal surgery. Am J Clin Nutr 2017; 106: 44-51. doi:10.3945/ajcn.116.148619

[43] McClave SA, Taylor BE, Martindale RG et al. Guidelines for the Provision and Assessment of Nutrition Support Therapy in the Adult Critically III Patient: Society of Critical Care Medicine (SCCM) and American Society for Parenteral and Enteral Nutrition (A.S.P.E.N.). JPEN J Parenter Enteral Nutr 2016; 40: 159-211. doi:10.1177/0148607115621863

[44] Gillis C, Buhler K, Bresee L et al. Effects of Nutritional Prehabilitation, With and Without Exercise, on Outcomes of Patients Who Undergo Colorectal Surgery: A Systematic Review and Meta-analysis. Gastroenterology 2018; 155: 391-410.e4. doi:10.1053/j.gastro.2018.05.012

[45] Moran J, Guinan E, McCormick P et al. The ability of prehabilitation to influence postoperative outcome after intra-abdominal operation: A systematic review and meta-analysis. Surgery 2016; 160: 1189-1201. doi:10.1016/j.surg.2016.05.014

[46] Bhatnagar S, Mittal A, Gupta SK et al. TWEAK causes myotube atrophy through coordinated activation of ubiquitin-proteasome system, autophagy, and caspases. J Cell Physiol 2012; 227: 1042-1051. doi:10.1002/jcp.22821

[47] Guttridge DC, Mayo MW, Madrid LV et al. NF-kappaB-induced loss of MyoD messenger RNA: possible role in muscle decay and cachexia. Science 2000; 289: 2363-2366. doi:10.1126/science.289.5488.2363

[48] Fukawa T, Yan-jiang BC, Min-Wen JC et al. Excessive fatty acid oxidation induces muscle atrophy in cancer cachexia. Nat Med 2016; 22: 666-671. doi:10.1038/nm.4093

[49] Fleck A, Raines G, Hawker F et al. Increased vascular permeability: a major cause of hypoalbuminaemia in disease and injury. Lancet 1985; 1: $781-784$

[50] Rozga ], Piatek T, Malkowski P. Human albumin: old, new, and emerging applications. Ann Transplant 2013; 18: 205-217. doi:10.12659/ AOT.889188

[51] Lindemann K, Kok PS, Stockler M et al. Enhanced Recovery After Surgery for Advanced Ovarian Cancer: A Systematic Review of Interventions Trialed. Int J Gynecol Cancer 2017; 27: 1274-1282. doi:10.1097| IGC.0000000000000981 